\section{Plasmon-induced CD response of oligonucleotide-conjugated metal nanoparticles $\dagger$}

\author{
Valérie A. Gérard, ${ }^{a}$ Yurii K. Gun'ko, ${ }^{* a}{ }^{a}$ Eric Defrancq ${ }^{b}$ and Alexander O. Govorov ${ }^{c}$ \\ Received 23rd February 2011, Accepted 7th May 2011 \\ DOI: $10.1039 / \mathrm{c} 1 \mathrm{cc11083g}$
}

Non-chiral metal nanoparticles conjugated with chiral oligonucleotide molecules demonstrated a circular dichroism (CD) at the plasmonic wavelengths due to aggregation effects.

Metal nanoparticles (NPs) have raised much interest in recent years due to their unique optical and electronic properties. Functionalising their surface with biomolecules has led the way to applications in fields as various as biosensing, ${ }^{1}$ drug delivery ${ }^{2}$ and Surface Enhanced Raman Spectroscopy (SERS). ${ }^{3}$ The induction or enhancement of a Circular Dichroism (CD) signal using metal nanoparticles (NPs) could be a new tool for highly responsive sensing. While biomolecules created by nature have a very high degree of homogeneity in a macroscopic ensemble and therefore produce amazingly strong and consistent CD signals (mostly in the UV range), it is still very challenging to create chiral nanocrystals because it seems impossible to control all positions of atoms in a nanocrystal. In many experiments, new $C D$ signals in the visible range in metal nanostructures were found for metal nano-clusters with a relatively small number of atoms and with the help of chiral molecules. ${ }^{4-8}$ An example is Ag clusters ( $\sim$ few hundreds of atoms) synthesized on DNA templates and giving new plasmonic CD lines. ${ }^{5}$ In few other experiments, plasmonic CD lines were found for conjugated NPs of larger sizes, having well-defined plasmonic lines. ${ }^{9-13}$

Suggested mechanisms of new CD bands in conjugated plasmonic nanocrystals include the formation of chiral surface states of the metal component, orbital interactions between the chiral adsorbate and the metal, and dipolar Coulomb interactions between chiral optical dipoles of conjugated molecules and plasmons. ${ }^{14}$ The latter mechanism was recently proposed theoretically ${ }^{14}$ and involves Coulomb interactions between nanoscale objects (chiral molecules and non-chiral nanocrystals), enabling plasmonic CD lines in the visible range. The main motivation for our research here was to create an artificial

${ }^{a}$ School of Chemistry, University of Dublin, Trinity College,

Dublin 2 Ireland.E-mail: igounko@tcd.ie

${ }^{b}$ Département de Chimie Moléculaire, UMR CNRS 5250, Université Joseph Fourier, BP 53, 38041 Grenoble cedex 9, France

${ }^{c}$ Department of Physics and Astronomy, Ohio University, Athens,

Ohio 45701,USA.E-mail: govorov@ohio.edu

$\dagger$ Electronic supplementary information (ESI) available: Experimental details on syntheses, DLS results, TEM images and spectra. See DOI: $10.1039 / \mathrm{c} 1 \mathrm{cc} 11083 \mathrm{~g}$ chirality coming from an interaction between well-defined nanoscale building blocks. In our work, we used assemblies of interacting chiral and non-chiral nanoscale elements, namely chiral oligonucleotide molecules and metal nanocrystals. Using initially non-chiral metal nanocrystals of various shapes, we first conjugated them with oligonucleotide molecules and then created aggregates of the new species. In a set of aggregated samples, we observed strong CD signals at the plasmonic wavelengths. It should be noted that aggregates of nanocrystals conjugated with non-chiral molecules did not show the optical chirality (CD signals). The plasmonic CD signals in aggregates composed of oligonucleotide-nanocrystal conjugates may come from the creation of hot plasmonic spots in aggregates and from the Coulomb interactions between chiral molecules and plasmons. Importantly, in contrast to many previous studies, the plasmonic CD mechanism found in this communication for chiral conjugated nanocrystals appeared only after aggregation. Additionally, breaking the aggregates using ultrasonic agitation leads to a reduction of the plasmonic $\mathrm{CD}$, which could be restored or increased by re-inducing aggregation. These data show the importance of collective plasmonic fields in the formation of chiral optical signals.

To start with a most simple system, spherical gold NPs were conjugated to oligonucleotides via their $3^{\prime}$ end T6 phosphorothioate groups. The conjugation of thiol-modified oligonucleotides to gold particles has been widely reported; however it usually involved a single terminal thiol group. ${ }^{1,15-18}$ Here, the affinity of the T6-phosphorothioate-modified oligonucleotide for gold NPs was assessed as a proof of concept before performing the conjugation with more highly shaped metal nanostructures. The UV-vis spectra of bare and oligonucleotide-conjugated particles showed a $7 \mathrm{~nm}$ red shift, from 519 to $526 \mathrm{~nm}$; this may be held as a first evidence of conjugation $^{1}$ as the red shift is induced by a dielectric function of the oligonucleotide shell (see ESI $\dagger$ ). The presence of oligonucleotides on the particle surface results in an increase in the size which was assessed by Dynamic Light Scattering (DLS) measurements. Bare gold NPs had an average hydrodynamic diameter of $20 \mathrm{~nm}( \pm 0.5 \mathrm{~nm})$ whereas that of conjugated particles was $70 \mathrm{~nm}( \pm 2 \mathrm{~nm})$. The oligonucleotide-conjugated spherical gold NPs did not show any major morphological or size differences compared to the original particles as observed using Transmission Electron Microscopy (TEM). 


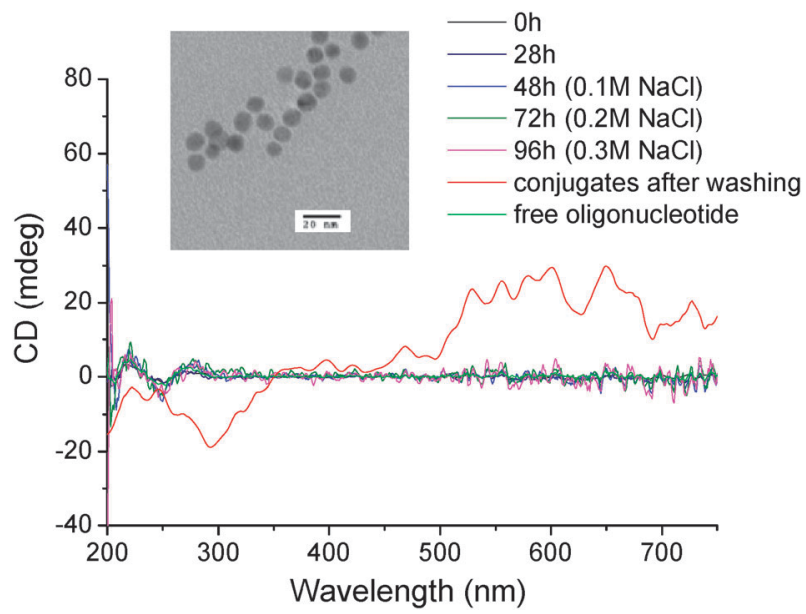

Fig. 1 Conjugation of oligonucleotides to spherical Au NPs monitored over time. Inset: TEM image of oligonucleotide-conjugated gold NPs.

Recently Govorov et al. demonstrated that non-chiral metal NPs coupled with surrounding chiral molecules (such as oligonucleotides and proteins) could enhance the CD signals of the chiral molecules as well as induce new CD signals in the plasmon region. ${ }^{14}$ To understand a physical mechanism for the appearance of the chiral plasmon peaks, we should note that the chiral molecules (oligonucleotides) typically do not absorb at the plasmonic wavelength (around $520 \mathrm{~nm}$ ), but they are optically-active and rotate light at this wavelength. Then, the plasmon in metal NPs "feels" a chiral non-absorbing environment created by the chiral molecules and becomes chiral. CD could thus be thought of as a potential technique to monitor the conjugation of oligonucleotides to gold NPs over time. However, the results shown in Fig. 1 indicate that gold NPs did not induce any significant change in the oligonucleotide CD signal when they were incubated together for up to four days with gradually increasing sodium chloride concentration. On the other hand, a strong CD signal appeared after washing the conjugates by centrifugation. The process caused partial aggregation of the particles as revealed by a $5 \mathrm{~nm}$ further red-shift in the plasmon band and confirmed by TEM. This observation can be qualitatively explained by the fact that aggregates typically have a stronger plasmon enhancement of electric fields inside due to the formation of hot spots in inter-NP junctions. ${ }^{19}$ This plasmon field enhancement should increase the interactions between external fields, chiral dipoles, and plasmons and, therefore, it should amplify a CD signal at the plasmonic wavelengths. ${ }^{14,19,20}$

Using the same method as previously described to conjugate oligonucleotides to spherical gold NPs, $3^{\prime}$ - phosphorothioatemodified oligonucleotides were bound to gold nanorings (NRs). The UV-Vis absorption spectrum of oligonucleotideconjugated gold NRs showed a $12 \mathrm{~nm}$ red shift associated with a broadening of the plasmon band compared to that of bare particles (see ESI $\dagger$ ). The peak broadening was attributed to partial aggregation of the NRs due to cross-linking of the oligonucleotides while the shift suggested effective conjugation manifested by a slight increase in particle size. This was further assessed by DLS measurement that showed an increase in the hydrodynamic radius (see ESI $\dagger$ ). Similarly to spherical NPs,

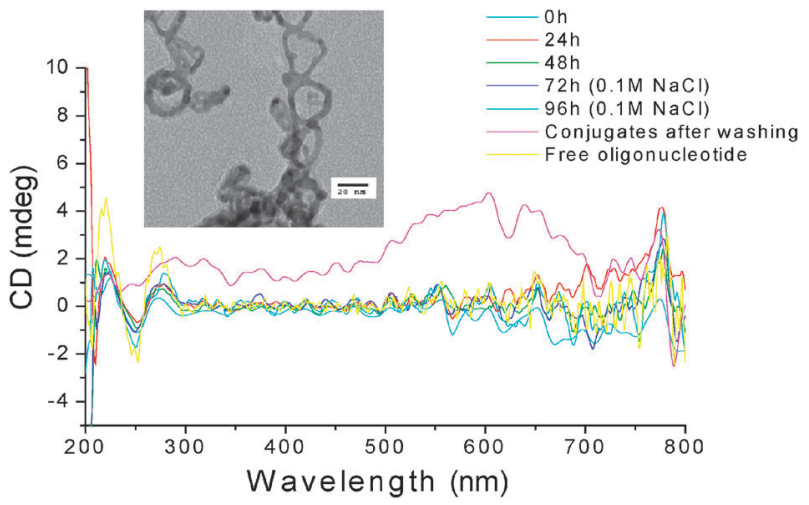

Fig. 2 Conjugation of oligonucleotides to Au NRs monitored over time and after washing. Inset: TEM image of oligonucleotide-modified gold NRs and CD spectra of conjugates over time and after washing.

the TEM images (ESI $\dagger$ ) did not show any significant difference in size and morphology between the bare and oligonucleotideconjugated gold nanorings. Finally, the CD response of the oligonucleotide-conjugated gold nanorings was monitored over time in the same way as for spherical particles and gave comparable results, as shown in Fig. 2; no significant change in the response was observed until after washing when a CD signal appeared in the plasmon resonance region which was attributed to plasmon-induced response from the oligonucleotide molecules in aggregates.

In order to verify that particle aggregation was not the only cause for the CD signal appearing after washing the conjugates, aggregation in the absence of chiral molecules was induced by increasing concentrations of sodium chloride. The size of thereby formed aggregates was monitored both by UV-Vis absorption spectroscopy and DLS (ESI $\dagger$ ). No plasmon induced signal was found for any of the aggregates, suggesting that they were not chiral. ${ }^{21}$ It could thus be assumed that the CD signal observed in the case of conjugates was related to the interaction between the chiral oligonucleotide molecules and the plasmonic particles.

To further assess the role of the plasmon-active species in the generation of $\mathrm{CD}$ signals, non-plasmonic particles, namely TGA-capped CdTe quantum dots (QDs), were conjugated to the oligonucleotides. The $5^{\prime}$ end amine function was used to covalently bind the oligonucleotide to a TGA molecule on the surface of the QDs via EDC coupling. The carboxylic acid group of TGA was first activated by using EDC for subsequent coupling reaction with the oligonucleotide terminal amine.

In contrast to metal nanoparticles these QD conjugates did not demonstrate any $\mathrm{CD}$ signal in the region between 450 and $750 \mathrm{~nm}$ (this region includes the exciton peaks) after washing followed by centrifugation (ESI $\dagger$ ), thus proving that plasmon resonance plays an essential role in the process. Indeed, we would qualitatively expect the chiral moleculeplasmon Coulomb interaction to be much stronger than the molecule-exciton coupling since the plasmon is much more optically active. We also note that chiral $\mathrm{CdS}$ and $\mathrm{CdSe}$ nanoparticles have been previously reported but in these nanostructures the chirality and emitting properties were induced by chiral surface defects. ${ }^{22-25}$

An important observation in this study is that the plasmoninduced CD band (500-700 $\mathrm{nm}$ ) appears for aggregated 


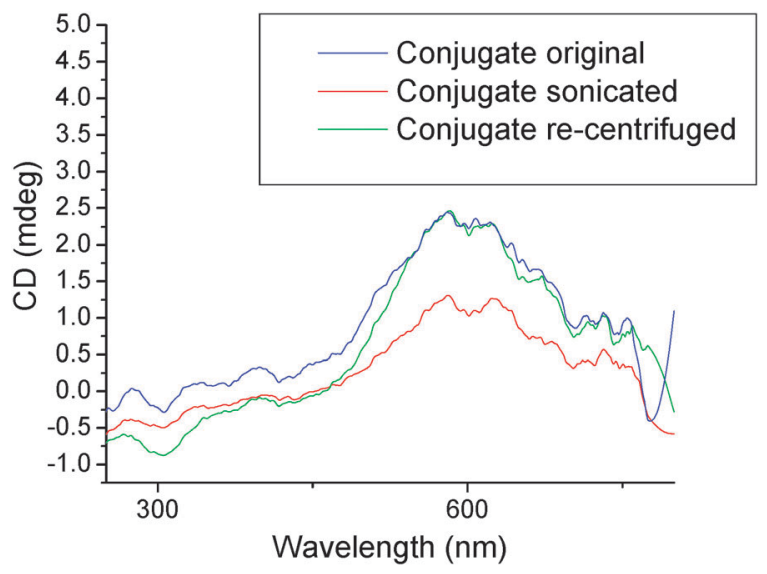

Fig. $3 \mathrm{CD}$ spectra of $\mathrm{Au}-\mathrm{NP}$ conjugates after centrifugation, sonication and second centrifugation.

nanocrystals of very different geometries (NPs and NRs). This observation is consistent with the Coulomb hot-spot mechanism since plasmonic hot spots will appear in both NP and NR systems. Looking more closely, we see that CD bands of NPs and NRs are quite similar. They form a broad spectral structure starting at $\sim 500 \mathrm{~nm}$ and ending at $\sim 700 \mathrm{~nm}$. As described above, we can interpret these $\mathrm{CD}$ structures as $\mathrm{CD}$ signals appearing due to Coulomb molecule-metal interaction in the plasmon hot-spot junctions between nanocrystals (NPs or NRs). These junctions (hot spots) contain greatly-enhanced electric fields which result in an enhancement of dynamic Coulomb interaction between chiral molecules and plasmons. Another remarkable observation, which is consistent with the Coulomb-interaction picture, is that the observed plasmon CD structures resemble very much the calculated spectra of the electric-field enhancement in the hot spot between two or several $\mathrm{Au}$ spheres, which were previously reported. ${ }^{26,27}$ The calculated spectra for enhancement factors have a broad structure spanning from 500 to $700 \mathrm{~nm}^{26,27}$

Finally an additional evidence of the importance of centrifugation was obtained by sonicating the $\mathrm{Au}-\mathrm{NP}$ conjugates to break the aggregates, which resulted in a reduced $\mathrm{CD}$; it could be restored to its original level by centrifuging again as shown in Fig. 3.

By contrast the intensity of Au-NR conjugates' $\mathrm{CD}$ bands did not decrease upon sonication $(\mathrm{ESI} \dagger)$. It seems that sonication did not break the NR based aggregates. Since the NRs are of different disk-like shape and thin (approx. $3 \mathrm{~nm}$ thick) their aggregates (e.g. stacks or sandwiches) are more stable and may not be disrupted by the short (30 s) sonication process. However, further centrifugation induced an increase in the CD intensity of the NR conjugates. Heating experiments on the nanoconjugates were also performed. We have found that heating of the conjugates from 20 to $40{ }^{\circ} \mathrm{C}$ did not have any pronounced effect on the intensity of $\mathrm{CD}$ signals. Only heating up to $50{ }^{\circ} \mathrm{C}$ resulted in some increase and red shift of $\mathrm{CD}$ signals indicating further aggregation of the conjugates due to their thermal decomposition (ESI $\dagger$ ).

In principle, $\mathrm{CD}$ signals at the plasmonic wavelength can appear due to chiral surface states created by a chiral adsorbate.
This interpretation for a CD signal at the plasmon wavelength was suggested by Katz et al. ${ }^{9}$ However, plasmonic CD signals in our system appeared only after aggregation that is thought to enhance the molecule-plasmon interactions. This suggests that the mechanism based on the Coulomb and electromagnetic interactions between chiral molecules and plasmons is a reasonable explanation for the observed plasmonic $\mathrm{CD}$ signals. Thus the new nanoconjugates demonstrated plasmon-induced $\mathrm{CD}$ signals from the oligonucleotide molecules in the plasmonic region of the NPs which is consistent with theoretical predictions. ${ }^{14}$ We believe that these new chiral nanomaterials could find a range of potential applications in new plasmonic devices and chiral sensing.

We acknowledge funding support from the Science Foundation Ireland (SFI), Trinity College Dublin (Ireland) and NSF (USA).

\section{Notes and references}

1 J. J. Storhoff, R. Elghanian, C. A. Mirkin, R. C. Mucic and R. L. Letsinger, J. Am. Chem. Soc., 1998, 120, 1959-1964.

2 K. Hamad-Schifferli, J. J. Schwartz, A. T. Santos, S. Zhang and J. M. Jacobson, Nature, 2002, 415, 152-155.

3 F. McKenzie and D. Graham, Chem. Commun., 2009, 5757-5759.

4 C. Gautier and T. Bürgi, ChemPhysChem, 2009, 10, 483-492.

5 T. Molotsky, T. Tamarin, A. B. Moshe, G. Markovich and A. B. Kotlyar, J. Phys. Chem. C, 2010, 114, 15951-15954.

6 J. George and K. G. Thomas, J. Am. Chem. Soc., 2010, 132, $2502-2503$.

7 V. Kitaev, J. Mater. Chem., 2008, 18, 4745-4749.

8 I. E. Santizo, F. Hidalgo, L. A. Perez, C. Noguez and I. L. Garzon, J. Phys. Chem. C, 2008, 112, 17533-17539.

9 J.-M. Ha, A. Solovyov and A. Katz, Langmuir, 2009, 25, 10548-10553.

10 J. M. Slocik, A. O. Govorov and R. R. Naik, Nano Lett., 2011, 11, 701-705.

11 P. Graf, A. Mantion, A. Haase, A. F. Thuünemann, A. Mašić, W. Meier, A. Luch and A. Taubert, ACS Nano, 2011, 5, 820-833.

12 H. S. Oh, S. Liu, H. Jee, A. Baev, M. T. Swihart and P. N. Prasad, J. Am. Chem. Soc., 2010, 132, 17346-17348.

13 P. Rezanka, K. Záruba and V. Král, Colloids Surf., A, 2010, 374, $77-83$.

14 A. O. Govorov, Z. Fan, P. Hernandez, J. M. Slocik and R. R. Naik, Nano Lett., 2010, 10, 1374-1382.

15 S. J. Hurst, A. K. R. Lytton-Jean and C. A. Mirkin, Anal. Chem., 2006, 78, 8313-8318.

16 R. C. Mucic, J. J. Storhoff, C. A. Mirkin and R. L. Letsinger, J. Am. Chem. Soc., 1998, 120, 12674-12675.

17 C. M. Niemeyer, B. Ceyhan, M. Noyong and U. Simon, Biochem. Biophys. Res. Commun., 2003, 311, 995-999.

18 J. J. Storhoff and C. A. Mirkin, Chem. Rev., 1999, 99, 1849-1862.

19 I. Lieberman, G. Shemer, T. Fried, E. M. Kosower and G. Markovich, Angew. Chem., Int. Ed., 2008, 47, 4855-4857.

20 A. O. Govorov, J. Phys. Chem. C, 2011, 115, 7914-7923.

21 Z. Fan and A. O. Govorov, Nano Lett., 2010, 10, 2580-2587.

22 M. P. Moloney, Y. K. Gun'ko and J. M. Kelly, Chem. Commun., 2007, 3900-3902.

23 S. D. Elliott, M. P. Moloney and Y. K. Gun'ko, Nano Lett., 2008, 8, 2452-2457.

24 S. A. Gallagher, M. P. Moloney, M. Wojdyla, S. J. Quinn, J. M. Kelly and Y. K. Gun'ko, J. Mater. Chem., 2010, 20, $8350-8355$.

25 J. E. Govan, E. Jan, A. Querejeta, N. A. Kotov and Y. K. Gun'ko, Chem. Commun., 2010, 46, 6072-6074.

26 A. O. Govorov, G. W. Bryant, W. Zhang, T. Skeini, J. Lee, N. A. Kotov, J. M. Slocik and R. R. Naik, Nano Lett., 2006, 6, 984-994.

27 F. J. Garcia de Abajo, J. Phys. Chem. C, 2008, 112, 17983-17987. 